\title{
Direct observation of antiferromagnetically oriented spin vortex states in magnetic multilayer elements
}

\author{
S. Wintz, ${ }^{1, a)}$ T. Strache, ${ }^{1}$ M. Körner, ${ }^{1}$ M. Fritzsche, ${ }^{1}$ D. Markó, ${ }^{1, b)}$ I. Mönch, ${ }^{2}$ R. Mattheis, ${ }^{3}$ \\ J. Raabe, ${ }^{4}$ C. Quitmann, ${ }^{4}$ J. McCord,${ }^{1}$ A. Erbe,${ }^{1}$ and J. Fassbender ${ }^{1}$ \\ ${ }^{1}$ Helmholtz-Zentrum Dresden-Rossendorf, 01328 Dresden, Germany \\ ${ }^{2}$ Institute for Integrative Nanosciences, IFW Dresden, 01069 Dresden, Germany \\ ${ }^{3}$ Institut für Photonische Technologien, 07702 Jena, Germany \\ ${ }^{4}$ SLS, Paul Scherrer Institut, 5232 Villigen, Switzerland
}

(Received 23 March 2011; accepted 15 May 2011; published online 9 June 2011)

\begin{abstract}
We report on the coupling of spin vortices in magnetic multilayer elements. The magnetization distribution in thin film disks consisting of two ferromagnetic layers separated by a nonmagnetic spacer is imaged layer-resolved by using x-ray microscopy. We directly observe two fundamentally different vortex coupling states, namely antiferromagnetic and ferromagnetic orientation of the flux directions. It is found that these states are predetermined for systems that involve a sufficiently strong interlayer exchange coupling, whereas for the case of a purely dipolar interaction both states are transformable into each other. (C) 2011 American Institute of Physics. [doi:10.1063/1.3597297]
\end{abstract}

Micromagnetism has been a focus of fundamental and technological research over several decades. Within this field, spin vortices have attracted much attention due to their chiral nature and the variety of dynamic phenomena that they exhibit. ${ }^{1-4}$ As illustrated in Fig. 1(a), such a spin vortex consists of a planar magnetization curl circulating around a nanometer sized core region, in which the magnetization turns perpendicular to the plane. ${ }^{1}$ They typically occur in ferromagnetic thin films, in particular as magnetic ground state of patterned micro- or nanostructures. ${ }^{5}$ In this kind of single layer structures, spin vortices have been observed directly_including their cores-using scanning probe ${ }^{6}$ and $\mathrm{x}$-ray microscopy. ${ }^{7}$ Their magnetodynamic properties depend on static topological charges, namely the winding number $n$ and the orientation of the core (polarity: $p$ ). ${ }^{2}$ For a common vortex $(n=1)$, the rotation sense of the gyrotropic core mode e.g., is fully determined by the $p$ value. ${ }^{2,3}$

When considering two or more vortices, interaction effects have to be taken into account. This holds true from the basic perspective, for instance the vortex-antivortex pair annihilation $^{8}$ as well as for potential applications such as magnetoresistive memory devices ${ }^{9}$ or vortex-based spintorque nano-oscillators. ${ }^{10}$ Of special interest in this technological context is the magnetic coupling between vertically stacked vortices, separated by a nonmagnetic spacer [cf. Fig. 1(b)]. Besides the dipolar interaction mediated by stray fields, interlayer exchange coupling (IEC) ${ }^{11}$ can be present in such magnetic multilayer systems.

The product of the in-plane flux direction (circulation: $c$ ) and the polarity $p$ defines the handedness $h=c p$ of a vortex. For a vortex pair this implies the existence of $2^{2}$ nondegenerate configurations, which can be categorized according to the combination of their individual quantum numbers $(p, h)$ or $(c, p) .{ }^{12}$ In the following we use $c \in\{-1,1\}$ and $p$ $\in\{-1,1\}$ to label the states as shown in Fig. 1(c). Equal vortex circulations $\left(\mathrm{FM} ; \Pi_{i} c_{i}=1\right)$ in the two layers correspond to a ferromagnetic ( $\mathrm{fm}$ ) orientation of the in-plane magnetization components while opposite circulations

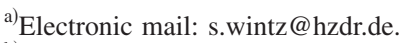

${ }^{b)}$ Present address: Université Paris-Sud 11, 91405 Orsay, France.
}

(AF; $\left.\Pi_{i} c_{i}=-1\right)$ correspond to an antiferromagnetic (afm) orientation. The core polarities $p_{i}$ in the two layers can be aligned either parallel $\left(\mathrm{P} ; \Pi_{i} p_{i}=1\right)$ or antiparallel (AP; $\left.\Pi_{i} p_{i}=-1\right)$. The four nondegenerate coupling states are expected to show different electrical resistances ${ }^{13}$ and qualitatively different dynamic responses to excitations by magnetic fields or spin-polarized currents. ${ }^{14-16}$ So far only stacked vortex pairs with FM configuration have been observed directly. ${ }^{17,18} \mathrm{AF}$ configurations have been only inferred indirectly, by comparing micromagnetic simulations with the results of integral magneto-optical ${ }^{19}$ and magnetoresistance ${ }^{12,13}$ measurements, respectively. On this background we report on the direct observation of interlayer coupled spin vortices with both FM and AF configurations, which we find in the presence of IEC and even for a purely dipolar coupling.

We studied three different trilayers as potential candidates for the formation of interlayer coupled vortices. Each trilayer consists of two ferromagnetic layers, namely cobalt (Co) and nickel ${ }_{81}$-iron $_{19}(\mathrm{NiFe})$, separated by a nonmagnetic spacer. Selecting different ferromagnetic elements allows the individual detection of the magnetic properties for each layer by element specific x-ray techniques. ${ }^{20}$ The thicknesses $(t)$ of the ferromagnetic layers are chosen so that for a micron sized lateral structure a magnetic vortex results as the ground state in each layer. ${ }^{21}$ A systematic variation of the interlayer cou-

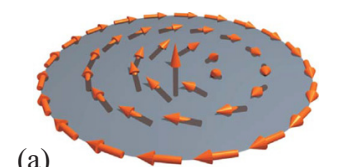

(a)



FM,P

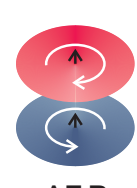

AF,P (b)
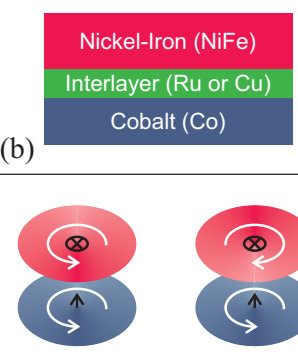

FM,AP



FIG. 1. (Color online) Illustration of magnetic disks and their micromagnetic structures. (a) Single layer disk with magnetization curl, indicated by arrows, and a vortex core in its center. (b) Multilayer stack, consisting of Co, a nonmagnetic interlayer, and $\mathrm{NiFe}$. (c) Four fundamental configurations with respect to the individual in-plane circulations ( $c$, white arrow) and core polarities ( $p$, black arrow) possible in a stack of two magnetic vortices. 

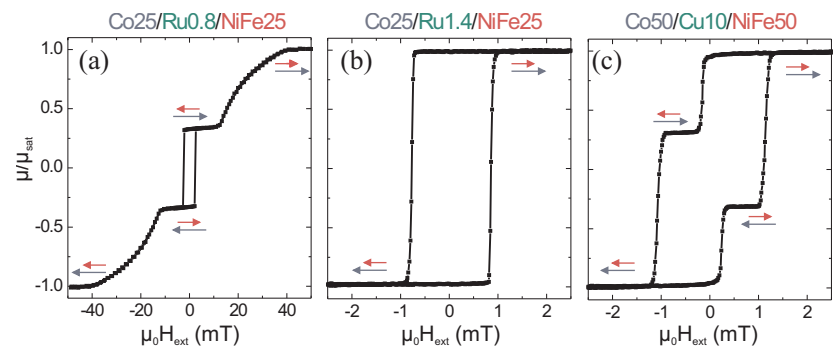

FIG. 2. (Color online) Magnetization reversal curves for three magnetic trilayer samples obtained by inductive magnetometry. (a) $\operatorname{Co}(23.9) / \mathrm{Ru}(0.8) /$ $\mathrm{NiFe}(21.4)(\mathrm{nm})$ sample with afm IEC showing a gradual reorientation of the NiFe layer followed by an abrupt switching of the entire stack at $2 \mathrm{mT}$. (b) $\mathrm{Co}(23.9) / \mathrm{Ru}(1.4) / \mathrm{NiFe}(21.4)(\mathrm{nm})$ film with fm IEC and simultaneous switching at $1 \mathrm{mT}$. (c) $\mathrm{Co}(47.8) / \mathrm{Cu}(10) / \mathrm{NiFe}(42.8)(\mathrm{nm})$ : dipolarly coupled layers showing independent switching of $\mathrm{NiFe}(0.25 \mathrm{mT})$ and $\mathrm{Co}(1 \mathrm{mT})$.

pling is achieved by using different thicknesses of ruthenium $(\mathrm{Ru})$ and copper $(\mathrm{Cu})$ as spacer layers. ${ }^{11}$ A ten target UHV system was used for the dc magnetron sputter deposition of continuous polycrystalline films with the following thicknesses: $\quad \mathrm{Co}(23.9) / \mathrm{Ru}(0.8) / \mathrm{NiFe}(21.4) / \mathrm{Al}(5), \quad \mathrm{Co}(23.9) /$ $\mathrm{Ru}(1.4) / \mathrm{NiFe}(21.4) / \mathrm{Al}(5)$, and $\mathrm{Co}(47.8) / \mathrm{Cu}(10) / \mathrm{NiFe}(42.8) /$ $\mathrm{Al}(3)(\mathrm{nm})$ onto thermally oxidized silicon wafers. Here the aluminum (Al) cap layer has a protective purpose only. The $\mathrm{Ru}$ thicknesses approximately correspond to the first minimum $\left(t_{\mathrm{Ru}}=0.8 \mathrm{~nm}\right.$, afm IEC) and maximum $\left(t_{\mathrm{Ru}}=1.4 \mathrm{~nm}\right.$, fm IEC) of the $t_{\mathrm{Ru}}$ dependent IEC oscillation. ${ }^{11}$ For the relatively thick $\mathrm{Cu}$ interlayer of $t_{\mathrm{Cu}}=10 \mathrm{~nm}$, a purely dipolar coupling is expected. In order to experimentally verify these different coupling types, magnetization reversal curves of the trilayers have been measured by inductive magnetometry.

Figure 2(a) shows the magnetization reversal loop of the $\mathrm{Co}(23.9) / \mathrm{Ru}(0.8) / \mathrm{NiFe}(21.4) \quad(\mathrm{nm})$ continuous film. The external field $\left(H_{\text {ext }}\right)$ is applied along the easy axis and in saturation both films are aligned parallel. When reducing the applied field a continuous $180^{\circ}$ reorientation of the $\mathrm{NiFe}$ layer to an antiparallel alignment occurs. This is supported by the relative magnetic moment $\mu / \mu_{\text {sat }}=0.33$ measured at remanence. Assuming standard material parameters ${ }^{22}$ for the saturation magnetization $\left(M_{\mathrm{Co}}=1440 \mathrm{kA} / \mathrm{m}, \quad M_{\mathrm{NiFe}}\right.$ $=800 \mathrm{kA} / \mathrm{m})$ one would expect a ratio of $\mu / \mu_{\mathrm{sat}}=\left(M_{\mathrm{Co}}\right.$ $\left.-M_{\mathrm{NiFe}}\right) /\left(M_{\mathrm{Co}}+M_{\mathrm{NiFe}}\right)=0.34$. At $\mu_{0} H_{\mathrm{ext}} \approx-2 \mathrm{mT}$ the whole stack undergoes a $180^{\circ}$ switching event. This is driven by the realignment of the stack's net-moment into the direction of the applied field while maintaining the antiparallel orientation of the layers. Beyond $\mu_{0} H_{\text {ext }} \approx-10 \mathrm{mT}$ the NiFe layer starts to rotate into the direction of the applied field again, gradually overcoming the afm IEC. Saturation, i.e., a parallel alignment of both layers, is reached at $\mu_{0} H_{\text {ext }} \approx-45 \mathrm{mT}$ $=\mu_{0} H_{\text {sat }}$. From the saturation field $\left(H_{\text {sat }}\right)$ we estimate the bilinear coupling constant to be $J \approx-0.5 \mathrm{~mJ} / \mathrm{m}^{2}$ using $J=$ $-\mu_{0} H_{\mathrm{sat}} M_{\mathrm{Co}} t_{\mathrm{Co}} M_{\mathrm{NiFe}} t_{\mathrm{NiFe}} /\left(M_{\mathrm{Co}} t_{\mathrm{Co}}+M_{\mathrm{NiFe}} t_{\mathrm{NiFe}}\right){ }^{23}$ This value confirms a strong afm IEC.

The film with an increased $\mathrm{Ru}$ thickness $\left(t_{\mathrm{Ru}}=1.4 \mathrm{~nm}\right)$ shows a simultaneous reversal of both layers at $\mu_{0} H_{\text {ext }}$ $\approx 1 \mathrm{mT}$ along the easy axis, thus indicating a fm IEC [cf. Fig. 2(b)]. The bilinear coupling strength cannot be derived from the shape of the hysteresis loop. ${ }^{23}$ Ferromagnetic resonance investigations ${ }^{24}$ however allow to determine an upper limit of $0.02 \mathrm{~mJ} / \mathrm{m}^{2}$ for $J$ (not shown). For the case of the $\mathrm{Cu}$ interlayer $\left(t_{\mathrm{Cu}}=10 \mathrm{~nm}\right)$ we observe two switching fields during the magnetization reversal along the easy axis [cf. Fig.

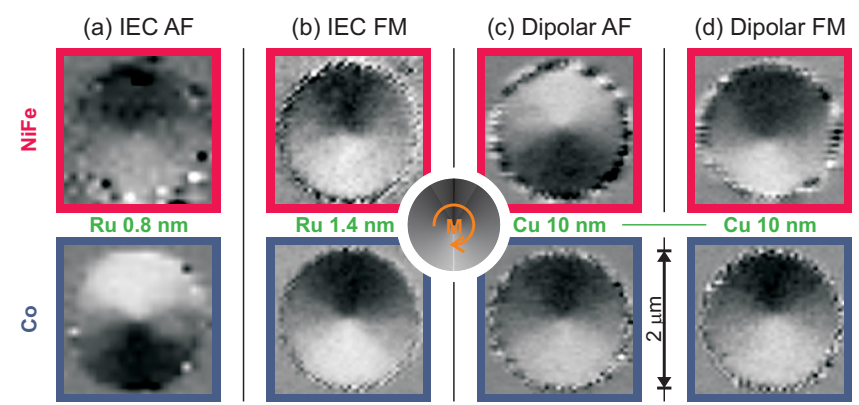

FIG. 3. (Color online) X-ray images showing the layer resolved magnetization for disks with $2 \mu \mathrm{m}$ diameter and varied interlayer coupling. Left columns: IEC (a) afm IEC $\left(t_{\mathrm{Ru}}=0.8 \mathrm{~nm}\right)$ leading to AF configurations of the vortices, (b) fm IEC $\left(t_{\mathrm{Ru}}=1.4 \mathrm{~nm}\right)$ resulting in FM circulation states. Right columns showing two examples for dipolarly coupled vortices $\left(t_{\mathrm{Cu}}\right.$ $=10 \mathrm{~nm}$ ) exhibiting both AF (c) and FM (d) configurations.

2(c)]. These events correspond to an initial switching of the relatively soft $\mathrm{NiFe}$ layer at $\mu_{0} H_{\mathrm{ext}} \approx 0.25 \mathrm{mT}$ and a subsequent switching of the more anisotropic Co layer at $\mu_{0} H_{\text {ext }}$ $\approx 1 \mathrm{mT}$, respectively. This independent switching behavior verifies the predicted purely dipolar coupling.

In order to confine magnetic vortices into a vertical arrangement, trilayer disks with diameters of $0.25,0.5,1$, and $2 \mu \mathrm{m}$ were fabricated onto silicon-nitride membranes using electron beam lithography, sputter deposition, and subsequent lift-off processing. After demagnetization, the distribution of the magnetization orientation $\mathbf{e}_{M}(\mathbf{r}):=\mathbf{M} / M$ in the disks was imaged using scanning transmission X-ray microscopy (STXM), for details see Ref. 25. This method provides a lateral resolution of about $25 \mathrm{~nm}$. A monochromatic X-ray beam is focused onto the sample by using a diffractive Fresnel zone plate. The sample itself is raster scanned through the beam while the transmitted intensity $(I)$ is measured. The application of circularly polarized x-rays at the element specific L-absorption edges leads to a magnetic contrast due to $\mathrm{x}$-ray magnetic circular dichroism. ${ }^{20}$ Here the local difference in transmissivity is proportional to the projection of the sample's local magnetization orientation onto the photon propagation direction $\left(\mathbf{e}_{k}\right) ; I \sim \mathbf{e}_{M} \mathbf{e}_{k}$. We therefore tilt the sample's normal by $30^{\circ}$ with respect to the beam axis in order to obtain information on the in-plane magnetization distribution. Layer selectivity is accomplished by imaging the sample with two different $\mathrm{x}$-ray photon energies, corre-

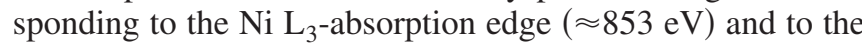
Co $\mathrm{L}_{3}$-absorption edge $(\approx 778 \mathrm{eV})$, respectively. This ensures that only the layer containing either of the elements contributes to the magnetic signal. The normalized difference of images taken with opposing circular light polarizations emphasizes the magnetic contrast while also removing topographic effects. As a reliable imaging of the relatively small vortex cores is at the detection limit of our setup in its present state, our investigation is focused on the vortex circulation configurations being AF or FM.

In the following we discuss the STXM results for interlayer coupled vortices in disks with a diameter of $2 \mu \mathrm{m}$. The magnetic contrast images of disks with different coupling are displayed in Fig. 3. From the continuous film measurements, afm IEC is expected for the disk containing a Ru spacer of $t_{\mathrm{Ru}}=0.8 \mathrm{~nm}$. In this disk we observe a vortex pair with opposite circulations, as can be seen in Fig. 3(a). Clearly the white region in the Co layer is located below a black region in the NiFe layer and vice versa. This means an AF configu- 
ration as sketched in Fig. 1(c) is realized. As this state is representative for most of the investigated structures of this trilayer type, we conclude that the afm IEC is sufficiently strong to deterministically induce the $\mathrm{AF}$ configuration. It is interesting to observe that the antiparallel magnetization orientation of both layers is strict on a local scale, which is a result of the relatively strong IEC. An increase of the Ru interlayer thickness to $t_{\mathrm{Ru}}=1.4 \mathrm{~nm}$ changes the IEC orientation to $\mathrm{fm}$ in the continuous film. A magnetic micrograph of a multilayer disk of this type is shown in Fig. 3(b). In contrast to Fig. 3(a), the same vortex circulation is exhibited in both layers, which corresponds to the FM configuration. For this structure we observe that the coupling is less strict; the position of the vortex core is slightly different in the two layers and the magnetization curl in the NiFe layer is not fully symmetric. Although the majority of the structures is in a micromagnetic state comparable to Fig. 3(b), we also observe a few FM vortex pairs whose cores are diametrically displaced from the center as well as nonvortex magnetization distributions in some rare cases. This situation can be partly attributed to the reduced strength of the IEC for larger Ru thicknesses. ${ }^{11}$ For vortices interacting only by dipolar coupling, we observe both circulation configurations FM and AF. Figures 3(c) and 3(d) show such disks whose magnetic layers are separated by a $\mathrm{Cu}$ spacer of $t_{\mathrm{Cu}}=10 \mathrm{~nm}$. While the magnetization distribution in Fig. 3(c) exhibits the AF configuration (opposite circulations), Fig. 3(d) shows a disk in the FM state (equal circulations). Here the configuration is not a fixed property of individual multilayer disks, e.g., induced by defects. Instead the configuration can be switched from $\mathrm{FM}$ to $\mathrm{AF}$ and back using demagnetization by an external field. However this switching is so far neither state selective nor deterministic. Again we also observe vortex pairs with diametrically off-centered core positions. At this point we cannot resolve if this is either due to pinning effects induced by sample imperfections or a consequence of lateral core repulsion that we anticipate for AP polarity configurations. States with $\mathrm{P}$ cores however, should favor centered and layer-congruent core positions assuming a lateral dipoledipole attraction of the cores. ${ }^{14}$ Note that we have investigated disks with different sizes from 0.25 to $2 \mu \mathrm{m}$ in diameter, though we find no qualitative changes with respect to lateral size for any of the three trilayers.

In summary we have investigated both patterned and continuous magnetic trilayer films. Hysteresis loops derived from inductive magnetometry provide information on the reversal process of continuous films and the type and strength of magnetic coupling involved. The local alignment of magnetization and the resulting vortex circulation configurations in multilayer disks are determined by layer-resolved x-ray microscopy. For IEC disks we directly observe and thereby prove the AF configuration, which had been predicted by others and we verify the FM configuration, already known to exist. These observations confirm the existence of fundamentally different vortex pair configurations in a vertical arrangement. For a thin $\mathrm{Ru}$ interlayer (strong afm IEC, AF states) the coupling is strict and local while for a thicker Ru interlayer (weak fm IEC, FM states) it is less strict. For disks with a purely dipolar coupling we observe both $\mathrm{AF}$ and FM con- figurations and switching between the two is possible using external magnetic fields. In the future, a better spatial resolution should allow the detection of the core polarity $p$ and the associated possible configurations. For disks with a purely dipolar coupling it should be possible to switch reliably between the FM and AF configuration using electrical currents or external fields, potentially leading to applications in data storage and spin-torque oscillators.

We thank A. Puzic, C. Bunce, and K. Lenz for their contribution to this work as well as V. Kühn, B. Eichler, B. Sarafimov, and K. Kirsch for technical support. The STXM experiments were performed using the X07DA (PolLux) beamline at the Swiss Light Source. Funding from BMBF (Contract Nos. 05 KS4WE1/6 and FKZ 13N10144) and EU (Grant No. 226716) is acknowledged.

${ }^{1}$ E. Feldtkeller and H. Thomas, Phys. Kondens. Mater. 4, 8 (1965).

${ }^{2}$ D. L. Huber, J. Appl. Phys. 53, 1899 (1982).

${ }^{3}$ S. B. Choe, Y. Acremann, A. Scholl, A. Bauer, A. Doran, J. Stöhr, and H. A. Padmore, Science 304, 420 (2004).

${ }^{4}$ B. Van Waeyenberge, A. Puzic, H. Stoll, K. W. Chou, T. Tyliszczak, R. Hertel, M. Fähnle, H. Brückl, K. Rott, G. Reiss, I. Neudecker, D. Weiss, C. H. Back, and G. Schütz, Nature (London) 444, 461 (2006).

${ }^{5}$ R. P. Cowburn, D. K. Koltsov, A. O. Adeyeye, and M. E. Welland, Phys. Rev. Lett. 83, 1042 (1999).

${ }^{6}$ T. Shinjo, T. Okuno, R. Hassdorf, K. Shigeto, and T. Ono, Science 289, 930 (2000).

${ }^{7}$ K. W. Chou, A. Puzic, H. Stoll, G. Schütz, B. Van Waeyenberge, T. Tyliszczak, K. Rott, G. Reiss H. Bruckl, I. Neudecker, D. Weiss, and C. H. Back, Appl. Phys. Lett. 90, 202505 (2007).

${ }^{8}$ R. Hertel and C. Schneider, Phys. Rev. Lett. 97, 177202 (2006).

${ }^{9}$ N. Kikuchi, S. Okamoto, O. Kitakami, Y. Shimada, S. Kim, Y. Otani, and K. Fukamichi, IEEE Trans. Magn. 37, 2082 (2001).

${ }^{10}$ A. Ruotolo, V. Cros, B. Georges, A. Dussaux, J. Grollier, C. Deranlot, R. Guillemet, K. Bouzehouane, S. Fusil, and A. Fert, Nat. Nanotechnol. 4 528 (2009).

${ }^{11}$ S. S. P. Parkin, Phys. Rev. Lett. 67, 3598 (1991).

${ }^{12}$ S. S. Cherepov, A. Konovalenko, V. Korenivski, and D. C. Worledge, IEEE Trans. Magn. 46, 2124 (2010).

${ }^{13}$ N. Locatelli, V. V. Naletov, J. Grollier, G. de Loubens, V. Cros, C. Deranlot, C. Ulysse, G. Faini, O. Klein, and A. Fert, Appl. Phys. Lett. 98, 062501 (2011)

${ }^{14}$ S.-H. Jun, J.-H. Shim, S.-K. Oh, S.-C. Yu, D.-H. Kim, B. Mesler, and P. Fischer, Appl. Phys. Lett. 95, 142509 (2009).

${ }^{15}$ K. Y. Guslienko, K. S. Buchanan, S. D. Bader, and V. Novosad, Appl. Phys. Lett. 86, 223112 (2005).

${ }^{16}$ A. V. Khvalkovskiy, J. Grollier, N. Locatelli, Y. V. Gorbunov, K. A. Zvezdin, and V. Cros, Appl. Phys. Lett. 96, 212507 (2010).

${ }^{17}$ J. Wu, D. Carlton, E. Oelker, J. S. Park, E. Jin, E. Arenholz, A. Scholl, C. Hwang, J. Bokor, and Z. Q. Qiu, J. Phys.: Condens. Matter 22, 342001 (2010).

${ }^{18}$ K. W. Chou, A. Puzic, H. Stoll, G. Schütz, B. Van Waeyenberge, T. Tyliszczak, K. Rott, G. Reiss, H. Bruckl, I. Neudecker, D. Weiss, and C. H. Back, J. Appl. Phys. 99, 08F305 (2006).

${ }^{19}$ K. Buchanan, K. Guslienko, A. Doran, A. Scholl, S. Bader, and V. Novosad, Phys. Rev. B 72, 134415 (2005).

${ }^{20}$ G. Schütz, W. Wagner, W. Wilhelm, P. Kienle, R. Zeller, R. Frahm, and G. Materlik, Phys. Rev. Lett. 58, 737 (1987).

${ }^{21}$ H. Hoffmann and F. Steinbauer, J. Appl. Phys. 92, 5463 (2002).

${ }^{22}$ R. C. O'Handley, Modern Magnetic Materials: Principles and Applications (Wiley, New York, 2000) p. 99.

${ }^{23}$ R. S. Beach, J. McCord, P. Webb, and D. Mauri, Appl. Phys. Lett. 80, 4576 (2002)

${ }^{24}$ J. Lindner and K. Baberschke, J. Phys.: Condens. Matter 15, S465 (2003)

${ }^{25}$ J. Raabe, G. Tzvetkov, U. Flechsig, M. Böge, A. Jaggi, B. Sarafimov, M. G. C. Vernooij, T. Huthwelker, H. Ade, D. Kilcoyne, T. Tyliszczak, R. H. Fink, and C. Quitmann, Rev. Sci. Instrum. 79, 113704 (2008). 